\title{
3. Neuromodulation for restoring memory
}

\author{
Sarah K. B. Bick, MD, and Emad N. Eskandar, MD \\ Department of Neurosurgery, Massachusetts General Hospital, Boston, Massachusetts
}

Disorders of learning and memory have a large social and economic impact in today's society. Unfortunately, existing medical treatments have shown limited clinical efficacy or potential for modification of the disease course. Deep brain stimulation is a successful treatment for movement disorders and has shown promise in a variety of other diseases including psychiatric disorders. The authors review the potential of neuromodulation for the treatment of disorders of learning and memory. They briefly discuss learning circuitry and its involvement in Alzheimer disease and traumatic brain injury. They then review the literature supporting various targets for neuromodulation to improve memory in animals and humans. Multiple targets including entorhinal cortex, fornix, nucleus basalis of Meynert, basal ganglia, and pedunculopontine nucleus have shown a promising potential for improving dysfunctional memory by mechanisms such as altering firing patterns in neuronal networks underlying memory and increasing synaptic plasticity and neurogenesis. Significant work remains to be done to translate these findings into durable clinical therapies.

http://thejns.org/doi/abs/10.3171/2016.3.FOCUS162

KEY WORDS deep brain stimulation; memory; Alzheimer disease; traumatic brain injury

$\mathrm{L}$ EARNING and memory are central to our core sense of self and to how we experience and interact with the world around us. Disorders of learning and memory such as dementia and traumatic brain injury (TBI) create a huge social and financial burden on society. Unfortunately, existing treatments have limited utility. Deep brain stimulation (DBS) is a successful treatment for movement disorders such as Parkinson disease (PD),${ }^{13}$ essential tremor, ${ }^{91}$ and dystonia, ${ }^{109}$ and more recently it has shown promise in a variety of other disorders including obsessive-compulsive disorder ${ }^{25}$ depression,${ }^{65}$ and Tourette syndrome. ${ }^{42}$ In this review we assess the use of DBS and other forms of neuromodulation to alter signaling in core nodes of the memory circuitry with the aim of improving dysfunctional learning and memory. We first briefly review the neural circuitry of memory and its involvement in Alzheimer disease (AD) and TBI, and then evaluate the evidence for reducing memory deficits through neuromodulation.

\section{Introduction to Memory}

\section{Anatomy of Memory Encoding and Retrieval}

The Papez circuit is part of the limbic circuitry and is integral to memory function (Fig. 1) ${ }^{80}$ It involves circular projections from hippocampus to fornix, to mammillary bodies, to anterior nucleus of the thalamus, to cingulum, to the entorhinal cortex, and back to the hippocampus. Much of the work on neuromodulation for memory has focused on altering activity in the Papez circuit.

The Papez circuit begins in the medial temporal lobe, an essential structure for new memory formation. Electrical stimulation of specific temporal lobe locations has been shown to evoke previous memories. ${ }^{102}$ The fimbria of the fornix receive projections from the hippocampus. The fornix has long been known to play an important role in memory, with lesions causing memory deficits. ${ }^{56}$ The precommissural fornix projects to the anterior cingulate cortex via septal nuclei and ventral striatum. ${ }^{38}$ The postcommissural fornix projects to the anterior nucleus of the thalamus and mammillary bodies..$^{23,38}$ The mammillary bodies project to the anterior nucleus of the thalamus via the mammillothalamic tract. The anterior nucleus of the thalamus provides glutamatergic input to the cingulate gyrus, which projects to the entorhinal cortex. ${ }^{38}$ The entorhinal cortex projects to the granule cell layer of the dentate gyrus and CA3 via the perforant pathway, as well as to subiculum and CA1 via the direct pathway, completing the circuit..$^{38,100}$ Dentate gyrus mossy fibers project to CA3, which projects to CA1 pyramidal neurons via Schaffer collaterals (Fig. 1). ${ }^{38}$

ABBREVIATIONS AD = Alzheimer disease; DBS = deep brain stimulation; GABA = $\gamma$-aminobutyric acid; MMSE = Mini-Mental State Examination; NBM = nucleus basalis of Meynert; NMDA = N-methyl-D-aspartate; $\mathrm{PD}=$ Parkinson disease; $\mathrm{PPN}=$ pedunculopontine nucleus; $\mathrm{STN}=$ subthalamic nucleus; $\mathrm{TBI}=$ traumatic brain injury. 


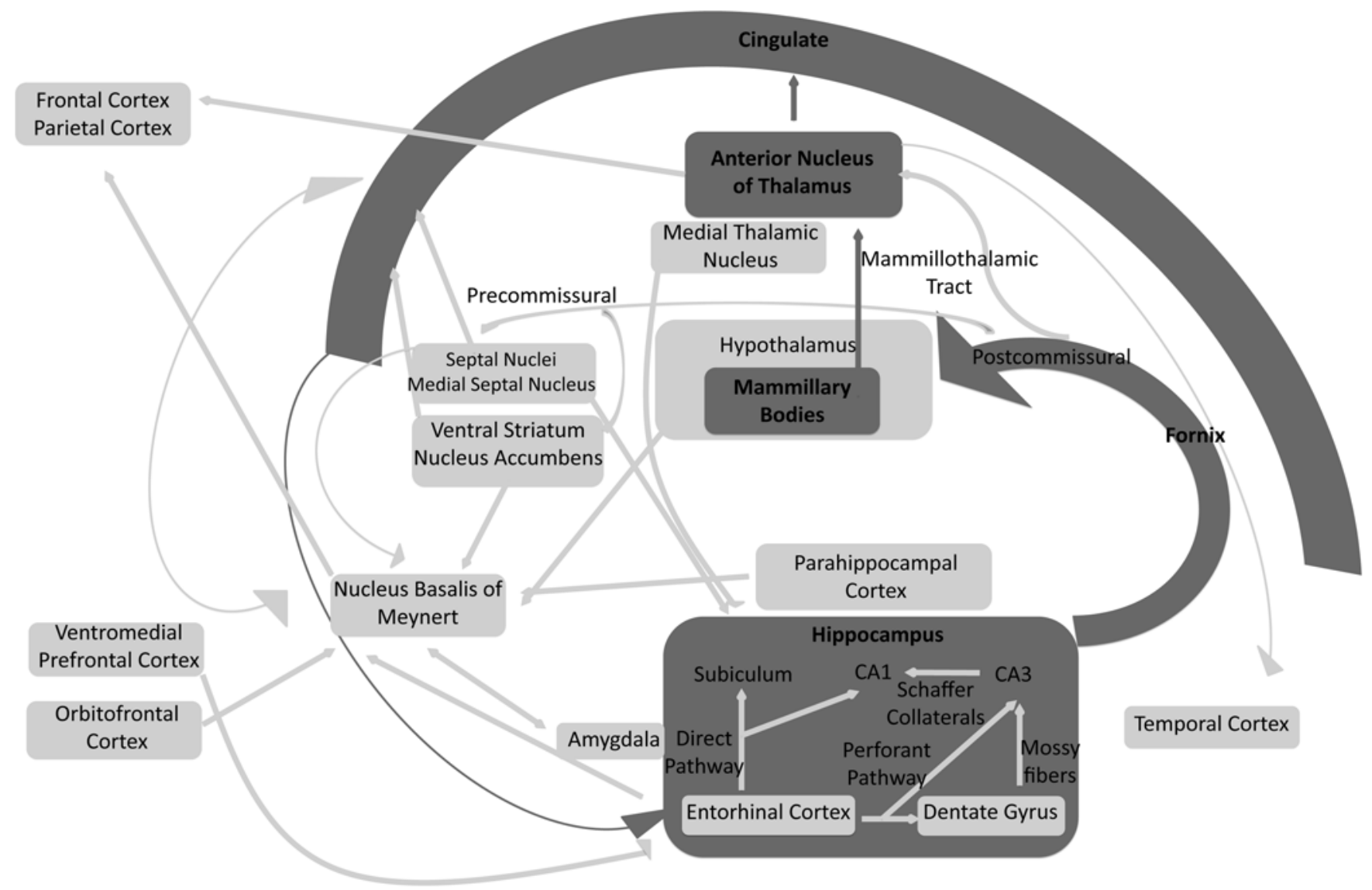

FIG. 1. Structures involved in memory circuitry and their interconnections. Dark gray structures indicate components of the circuit of Papez, and light gray structures indicate other components of memory circuitry discussed in the text. Memory circuitry is highly distributed and interconnected.

In addition to particular anatomical regions, specific firing patterns have been implicated in memory functions. Groups of neurons influence the activity of other surrounding neurons, generating different frequency oscillations in extracellular field potentials recorded via local field potentials or electroencephalography. ${ }^{16}$ These oscillations can occur at a wide range of frequencies. Two frequencies that play a particularly important role in memory are gamma $(30-100 \mathrm{~Hz})$ and theta $(3-8 \mathrm{~Hz})$. Theta-frequency oscillations predominate hippocampal activity and play a vital role in memory, in part by contributing to the transfer of information between the hippocampus and other regions involved in memory..$^{58}$ Phase synchronization occurs when the oscillation patterns of 2 connected regions become coordinated, and plays an important role in communication between neural regions as well as neural plasticity. Because of its higher frequency, gamma-phase synchronization plays an important role in neural communication and long-term potentiation by providing a tight temporal regulation and coordination of neural signals. Theta-phase synchronization also plays an important role in neural communication and plasticity processes contributing to memory, perhaps partially by providing a more globalscale coordination of local gamma-phase synchronized assemblies. ${ }^{16}$ Gamma-phase synchronization between the rhinal cortex and hippocampus has been implicated in both successful memory encoding and retrieval. Rhinal cortex and hippocampus theta-phase synchronization occurs during memory encoding, whereas medial temporal lobe theta-phase synchronization with neocortical regions is increased during memory retrieval..$^{59}$ Anterior thalamic nucleus-frontal lobe and parietal lobe theta synchrony is seen during successful memory encoding. ${ }^{104}$ An interconnected network of cortical and subcortical regions referred to as the default mode network underlies memory function. In healthy young adults, increased connectivity is seen in the resting state, with decreased connectivity during cognitive tasks. ${ }^{62}$

\section{Types of Memory}

Memory can be divided into short- and long-term memory. Short-term or working memory involves temporarily holding and manipulating information for cognitive tasks. Long-term memory is a system that allows more permanent storage, management, and retrieval of information. Long-term memory can be divided into implicit, or unconscious recall, and explicit, or conscious recall. Implicit or procedural memory is a type of memory in which associations are formed between environmental sensory cues and behavioral responses ${ }^{43}$ Explicit memory can be subdivided into semantic memory, which involves retaining facts independent of environmental setting, and episodic 
memory, which refers to the memory of autobiographical events. ${ }^{43}$ Other types of memory include spatial memory, which involves information about the relationship of objects to the spatial environment.

Different types of memory rely on different components of memory circuitry. Episodic memory heavily uses the hippocampus and anterior nucleus of the thalamus. ${ }^{38}$ The hippocampus, anterior nucleus of the thalamus, parahippocampal gyrus, and entorhinal cortex are important in semantic memory. ${ }^{38}$ The hippocampus and anterior nucleus of the thalamus also play a role in spatial memory, as do the fornix, cingulum, parahippocampal gyrus, and entorhinal cortex.

\section{Disorders of Learning and Memory}

\section{Alzheimer Disease}

Alzheimer disease is the most common dementia and is associated with tremendous social and economic costs. ${ }^{38}$ The prevalence of AD in the US is $9.7 \%$ in patients older than 70 and increases to $37.4 \%$ in those older than 90.84 The prevalence of AD in the US is 5.2 million and is expected to increase significantly in coming years. ${ }^{31,36}$

Alzheimer disease is characterized by cognitive deterioration, memory loss, aphasia, apraxia, and behavioral symptoms. ${ }^{84}$ Difficulty with learning new information, formation of episodic memory, memory recall, and problem solving are characteristic. ${ }^{59}$ Numerous cerebral amyloid plaques made up of aggregated beta amyloid peptides and intracellular neurofibrillary tangles composed of hyperphosphorylated tau protein are found in the brains of patients with AD. ${ }^{33}$

Alzheimer disease is associated with decreased metabolism in frontal lobe, medial temporal lobe, and parietal regions, and is characterized by hippocampal cell loss, degeneration of the basal forebrain cholinergic nucleus basalis of Meynert (NBM), and dysfunction of the noradrenergic locus coeruleus and serotonergic dorsal raphe nucleus (Fig. 2). ${ }^{38}$ Atrophy of entorhinal cortex and hippocampus may occur before the development of cognitive symptoms. Forniceal degeneration has also been associated with early cognitive impairment. ${ }^{62}$

Dysfunctional neural networks with metabolic abnormalities across wide cortical and subcortical regions contribute to the deficits seen in AD. ${ }^{55,92}$ Deactivation in the default mode network during cognitive tasks is decreased in older adults and in patients with $\mathrm{AD}$, with greater deficits in the latter. ${ }^{62}$ In AD there is decreased activity in the precuneus and posterior cingulate, which comprise the posterior default mode network, with decreased connectivity in all areas as the disease progresses ${ }^{83}$ Current treatment focuses on cholinesterase inhibitors and memantine (an $\mathrm{N}$-methyl-D-aspartate [NMDA] receptor antagonist), but unfortunately has limited efficacy. ${ }^{33,84}$

\section{Traumatic Brain Injury}

Traumatic brain injury is a major cause of death and disability in the US..$^{14,81}$ The annual incidence of TBI in the US is approximately 1.5 million. Initial damage occurs due to direct physical damage to the brain, with further damage occurring due to secondary processes such as glutaminergic excitotoxicity, disruption of the blood-brain barrier, and increased intracranial pressure. Cognitive deficits after TBI include impairments in attention, concentration, information processing, memory, and problem solving..$^{14}$

Traumatic brain injury reduces theta-frequency firing, a neural firing frequency known to play an important role in memory, in rat hippocampus CA1, ${ }^{77,58}$ as well as firing in other frequency ranges. TBI decreases broadband power as well as theta oscillations in the hippocampus, perhaps associated with a change in the level of neuronal correlation. ${ }^{82}$ Also, TBI has been associated with increased neuronal and network excitability. ${ }^{30}$

\section{Targets for Neuromodulation Cortical Targets \\ Entorhinal Cortex}

Entorhinal cortex stimulation in rodents increases neurogenesis in the dentate gyrus and improves spatial working memory in a manner dependent on neurogenesis. ${ }^{100}$ Adult-generated dentate gyrus cells are incorporated into hippocampal memory networks. ${ }^{101}$

Stimulation of entorhinal cortex during learning of a spatial memory task enhanced learning in human subjects with electrodes implanted for evaluation of treatment-resistant epilepsy, whereas hippocampal stimulation did not affect performance. Entorhinal stimulation also increased hippocampal theta power, the firing frequency known to be important for the role of the hippocampus in memory ${ }^{103}$ Entorhinal cortex stimulation may improve memory encoding by augmenting hippocampal theta activity as well as having long-term benefits on memory capacity by increasing production of new neurons integrated into functional hippocampal memory circuitry.

\section{Alzheimer Disease Pathology}

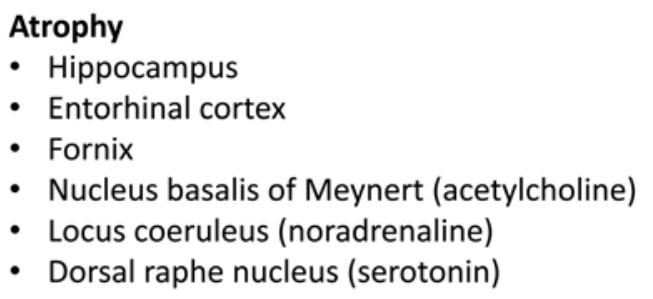

Decreased Metabolism

- Frontal

- Medial temporal

- Parietal

- Cingulate
Connectivity

- Dysfunctional neural networks across wide cortical and subcortical regions

- Decreased connectivity default mode network

FIG. 2. Summary of pathological changes involved in $A D$. 


\section{Hippocampus}

The hippocampus is integral to the creation of new memories but not to memory storage ${ }^{4} \mathrm{CA} 3$ and dentate gyrus are important in memory encoding. ${ }^{102}$ Hippocampal dysfunction with anterograde amnesia is characteristic of both $\mathrm{AD}$ and TBI. ${ }^{4}$ Degeneration of hippocampal neurons may be an early event in the pathogenesis of AD. ${ }^{38}$

Hippocampal neuronal recording and activation has been used to record and generate memories in rodents. When neurons activated during fear learning are later activated using optogenetic stimulation in the context of the conditioned stimulus, animals display increased fear response, indicating memory activation. ${ }^{61}$ Recording ensembles of hippocampal CA1 and CA3 neurons enable prediction of memory task outcome, and stimulation alters memory task performance. ${ }^{5}$ Hippocampal stimulation delivered in a spatial and temporal task-specific pattern determined from prior recordings restored memory-dependent conditioned behavior in rats treated with hippocampal infusion of an NMDA channel blocker, a treatment that blocks hippocampal-dependent memory performance. ${ }^{4}$

Medial temporal DBS has shown some promise in a rodent dementia model. Scopolamine is a muscarinic acetylcholine receptor antagonist, and animals treated with this agent have memory impairment, similar to patients with AD who have a cholinergic deficit. Entorhinal cortex and CA1 stimulation during a spatial memory task restored performance in a rat scopolamine injection dementia model ${ }^{37} \mathrm{CA} 1$ stimulation increased expression of a neuronal activity marker in the cingulate gyrus, mammillothalamic tract DBS increased levels in the infralimbic and prelimbic cortex, and entorhinal cortex and anterior thalamic nucleus stimulation increased levels in CA3. ${ }^{37}$

Much of the hippocampal stimulation research in human subjects has been performed in patients with epilepsy-with mixed results. Stimulation of medial temporal structures including the hippocampus and amygdala may evoke visual memories in human subjects. ${ }^{110}$ However, bilateral hippocampal DBS for epilepsy may cause memory impairments. ${ }^{66}$ Epilepsy patients with implanted depth electrodes who underwent bilateral hippocampal stimulation during encoding in an episodic memory task had impaired performance on the task, whereas stimulation during retrieval did not affect performance. ${ }^{54}$ Stimulation may cause a depolarization block, which disrupts formation of episodic memory. ${ }^{38}$ Dominant-side hippocampal DBS may have detrimental effects on verbal intelligence. ${ }^{70}$ Conversely, a group of epilepsy patients who underwent unilateral amygdalohippocampal DBS had improved visual memory (which may have been due to a practice effect), but otherwise had unchanged cognitive performance. Overall, direct hippocampal DBS in humans does not appear to improve memory, and bilateral stimulation may alter hippocampal activity that is necessary for memory encoding and thus impair memory formation.

Gamma oscillation synchronization between rhinal cortex and hippocampus is associated with successful memory encoding. ${ }^{17}$ In epilepsy patients with implanted depth electrodes who received coordinated hippocampal and rhinal cortex stimulation during a learning task, the phase of stimulation affected performance, with in-phase, sham, and anti-phase stimulation, respectively, having decreasing efficacies. ${ }^{17,18}$ Overall, these studies confirm that the hippocampus is important for memory encoding and suggest that sophisticated stimulation parameters or stimulation of multiple regions to increase connectivity may be necessary to recruit hippocampal function and improve memory through neuromodulation.

\section{Neocortical Regions}

The ventromedial prefrontal cortex has indirect connections with the hippocampus; thus, strategies targeting this region may improve memory by augmenting hippocampal function. ${ }^{60}$ In rats, left medial prefrontal cortex epidural direct-current stimulation prior to a spatial working memory task improved performance. The medial prefrontal cortex may contribute to learning strategies involved in working memory. ${ }^{12}$

In a rat aging model of dementia, older rats have deficits in long-term memory. Both high- and low-frequency stimulation of the ventromedial prefrontal cortex prior to training and testing improved short- but not long-term memory, whereas chronic stimulation improved both short- and long-term memory. High-frequency stimulation increased a marker of neural activity in subiculum, dentate gyrus, and CA1, and it increased the expression of genes related to neurogenesis, as well as increasing neural progenitor cells and dendritic spines in the dentate gyrus. Improvements in spatial memory were associated with cell proliferation in the dentate gyrus. ${ }^{60}$

Prefrontal cortex plays an important role in memory encoding and retrieval, and increased dorsolateral prefrontal cortex activity has been demonstrated in AD. ${ }^{31}$ Anodal transcranial direct-current stimulation of dorsolateral prefrontal cortex during encoding, which is known to be activating, improved performance on a verbal memory task. Conversely, cathodal stimulation during encoding, which is deactivating, impaired performance. When the stimulation was performed during recall, anodal stimulation trended toward improved verbal memory performance, whereas cathodal stimulation impaired performance. ${ }^{46}$ This suggests that dorsolateral prefrontal cortex activation improves both verbal memory encoding and retrieval, whereas decreased activity impairs performance.

Repetitive transcranial magnetic stimulation of the left dorsolateral prefrontal cortex in patients with postconcussive syndrome resulted in improvement in symptoms, including subjective improvement in memory, and was associated with greater dorsolateral prefrontal cortex activity and reduced rostral anterior cingulate cortex activity during a working memory task. ${ }^{52}$ Cortical activity induced by left prefrontal cortex anodal transcranial direct-current stimulation in patients with TBI correlated with improvements on neuropsychological tests. ${ }^{108}$

Anodal transcranial direct-current stimulation of prefrontal cortex improves working memory. ${ }^{21}$ Transcranial magnetic stimulation of left inferior prefrontal cortex was associated with improved episodic memory. ${ }^{51}$ In a group of elderly patients who reported memory loss, prefrontal repetitive transcranial magnetic stimulation improved performance on an associative memory task, and was associated with increased activity in the left anterior cin- 
gulate and right middle frontal gyrus and in the medial frontal lobe. ${ }^{96}$

Stimulation of parietal cortex has also been associated with memory improvements. In patients with mild cognitive impairment, repetitive transcranial magnetic stimulation of parietal cortex improved associative memory. ${ }^{11}$ Transcranial alternating-current stimulation of the left parietal cortex during a working memory task increased working memory storage capacity. ${ }^{45}$

Given the known importance of the temporal lobe in memory function, temporal lobe stimulation has been explored for memory modulation. A case report describing a woman undergoing temporoparietal deep transcranial magnetic stimulation for treatment of tinnitus found that pleasant childhood memories were evoked in the patient by stimulation, ${ }^{87}$ suggesting that it may be possible to leverage stimulation of this region to augment memory recall. Noninvasive temporal stimulation has shown promise in both $\mathrm{AD}$ and TBI. Temporal anodal direct-current stimulation in a group of patients with $\mathrm{AD}$ improved performance on a visual recognition memory task, with effects lasting for up to 1 month after stimulation. ${ }^{6}$ Left frontotemporal repetitive transcranial magnetic stimulation in a patient with TBI resulted in improvement in executive functions and memory. ${ }^{79}$ Larger efforts to follow up on these reports are warranted.

\section{Subcortical Targets}

\section{Anterior Nucleus of the Thalamus}

The anterior nucleus of the thalamus is part of the $\mathrm{Pa}$ pez circuit and has connections with a number of areas known to be involved in memory. It projects to both superior frontal and temporal cortical regions ${ }^{19}$ as well as to the dentate gyrus via the cingulum and entorhinal cortex, ${ }^{27}$ and has strong connections with the anterior cingulate cortex..$^{35}$ It receives projections from mammillary bodies via the mammillothalamic tract, and from the subiculum via the fornix; ${ }^{3}$ therefore it is well positioned to modulate memory.

High-frequency stimulation of the anterior nucleus of the thalamus improves spatial memory in a mouse AD model. ${ }^{9}$ Rats treated with corticosterone to reduce neurogenesis have impaired performance on a hippocampal memory task, but performance returned to baseline and hippocampal neurogenesis increased in rats that received high-frequency stimulation of the anterior thalamic nucleus 1 month prior to testing. ${ }^{29}$ High-frequency but not lowfrequency stimulation of the anterior thalamic nucleus increased neurogenesis in the dentate gyrus, ${ }^{15,106}$ with a large portion of new cells developing into mature neurons, and high-frequency stimulation also restored decrements in dentate gyrus neurogenesis induced by corticosteroid treatment ${ }^{106}$ Conversely, high-current-density stimulation of anterior thalamic nucleus during memory acquisition impaired performance on the memory task and resulted in increased markers of neural activity in cingulate cortex, orbitofrontal cortex, supramammillary nucleus, and lateral entorhinal cortex, and in a decreased firing rate of dentate gyrus cells in vivo. ${ }^{27}$

In a double-blinded randomized controlled trial of bi- lateral DBS of the anterior thalamic nucleus for epilepsy, patients with stimulation turned on reported a greater incidence of subjective memory impairment, although no difference was observed on neuropsychological cognitive testing. ${ }^{19}$ Patients with epilepsy who received DBS of the anterior thalamic nucleus had worsened response inhibition and enhanced attention allocation to threat with stimulation turned on than with it turned off. ${ }^{35}$ In another study, patients with bilateral DBS of the anterior thalamic nucleus had improved word fluency and verbal memory, which did not correlate with seizure reduction..$^{77}$ Observed differences may be related to differences in stimulation parameters, timing of stimulation delivery, and timing of behavioral testing. Given the role of the anterior thalamic nucleus in the Papez circuit and demonstrated changes in activity in regions involved in memory with anterior thalamic nucleus stimulation, DBS of this region may impact memory by Hebbian learning, strengthening connections between regions involved in learning circuitry. Animal models have also demonstrated changes in neurogenesis with anterior thalamic nucleus stimulation; thus some of the differences in effects seen in human subjects with DBS of this region may be related to timing of behavioral testing, because time between initiation of DBS and behavioral testing is necessary to allow for neurogenesis to occur if this mechanism contributes to the effects seen in human subjects. Further trials will be required to determine if the promise of this therapeutic target in rodent models translates to human patients, and to further elucidate the mechanism of action.

\section{Other Thalamic Regions}

The rostral intralaminar thalamic nuclei are part of frontal basal ganglia networks and play a role in maintaining arousal. Low-current stimulation of rostral intralaminar thalamic nuclei in rats just prior to memory retrieval improved performance on a delayed match-tosample task, whereas high-current stimulation impaired it, suggesting that activation of this structure is involved in memory retrieval. ${ }^{63}$

Midline thalamic nuclei have connections with the hippocampus, and stimulation may result in activation of CA1. Medial thalamic nucleus high-frequency stimulation increased alpha but not beta secretase activity in an AD mouse model. Medial thalamic nucleus stimulation in a mouse AD model improved acquisition memory and short-term memory, and increased activity in CA1 and dentate gyrus. These findings suggest that medial thalamic nucleus high-frequency stimulation may enhance synaptic plasticity and alter A-beta clearing. ${ }^{2}$

Central thalamus high-frequency stimulation increased expression of a marker of neuronal activity as well as a gene associated with long-term potentiation in motor cortex, anterior cingulate cortex, and dentate gyrus. Stimulation during the memory recall period but not during the learning or interval period improved performance on a novel object recognition task. ${ }^{93}$

Stimulation of left but not right ventrolateral thalamus in human subjects during memory recall impaired performance on a verbal memory test, whereas stimulation during both presentation and recall did not impair per- 
formance. Also, stimulation of left ventrolateral thalamic electrodes during stimulus presentation was associated with improved performance, suggesting that the right ventrolateral thalamus may contribute more to memory recall and the left ventrolateral thalamus may contribute more to memory encoding. ${ }^{78}$

\section{Hypothalamus}

The lateral hypothalamus has connections with the medial forebrain bundle and other components of the reward system; stimulation is rewarding and reinforces learning. Rats with amygdala lesions, which are known to have learning and memory deficits, have improved performance with lateral hypothalamus self-stimulation following a fear-learning task. This improvement is associated with decreased acetylcholinesterase activity in preserved lateral amygdala areas. ${ }^{49}$

A patient who underwent ventral hypothalamic stimulation for obesity reported that the stimulation induced autobiographical memories, and the patient had improved performance on verbal learning and spatial associative learning tasks as well as a hippocampal-mediated memory task with stimulation. Hypothalamic stimulation drove increased activity in the ipsilateral hippocampus and parahippocampal gyrus, probably via activation of the fornix..$^{28}$

\section{Medial Septal Nucleus}

The medial septum has cholinergic, $\gamma$-aminobutyric acid (GABA)ergic, and glutaminergic projections to the hippocampus. Theta-frequency activity dominates hippocampal neural firing patterns and is known to play an important role in memory. $16,57,58$ Connections between the medial septal nucleus and hippocampus have an important role in regulating hippocampal theta oscillations, ${ }^{111}$ with the medial septal nucleus acting as a pacemaker for the hippocampus. ${ }^{58}$ As such, the medial septal nucleus is positioned to modulate hippocampal activity and memory function. ${ }^{47}$

Medial septal nucleus stimulation prior to a spatial working memory task increased hippocampal theta oscillations $\mathrm{s}^{58}$ and improved spatial working memory in a TBI rat model, but did not have a significant effect in control rats. ${ }^{57}$ Theta-frequency stimulation during a spatial learning task in a rat TBI model also improved learning. ${ }^{58} \mathrm{Me}-$ dial septal nucleus stimulation therefore appears to augment hippocampal theta oscillations, and may improve memory encoding by strengthening this rhythm, with its known importance in memory.

A study in a cholinergic lesion rat model of dementia found that medial septum midfrequency stimulation performed daily following training sessions improved learning in a spatial memory task and increased hippocampal acetylcholinesterase activity and dentate gyrus neurogenesis. ${ }^{47}$ Therefore increased medial septal nucleus stimulation may also improve memory via increased hippocampal activity and neurogenesis.

\section{Nucleus Basalis of Meynert}

Both AD- and PD-related dementia are characterized by loss of cholinergic neurons in the NBM as well as loss of cortical cholinergic markers. ${ }^{24,33}$ The NBM modulates widespread cortical networks involved in cognitive func- tion. ${ }^{24}$ It is a largely cholinergic nucleus providing cholinergic innervation to cortex, which modulates other cortical inputs. ${ }^{33}$ It receives projections from limbic and paralimbic regions including orbitofrontal cortex, parahippocampal cortex, entorhinal cortex, and cingulate cortex, as well as amygdala, hypothalamus, septal nuclei, and nucleus accumbens. The NBM has widespread neocortical projections, including frontal, parietal, and cingulate cortex as well as amygdala. Cortical cholinergic function is required for the formation of new memories, and the NBM is known to be involved in cortical plasticity and electrophysiological representation of learning and memory. ${ }^{24}$

Stimulation of the NBM has been shown to induce an associative memory in an auditory learning task in healthy rats. ${ }^{67}$ Low-frequency DBS of the NBM in healthy rats prior to training improved memory acquisition, whereas stimulation after training did not affect retention, suggesting that NBM DBS may enhance memory encoding. ${ }^{73}$ Stimulation of the NBM has been shown to increase cortical acetylcholine release in a rodent model ${ }^{85}$ therefore this may occur via enhanced cholinergic release, which alters plasticity. ${ }^{34,73,74}$ Unilateral low-frequency NBM stimulation prior to training improved memory acquisition and increased expression of a marker of neural activity in orbitofrontal, prelimbic, and infralimbic cortices, and the hippocampal subregions dorsal CA and ventral dentate gyrus. ${ }^{7}$ NBM stimulation paired with an auditory tone results in reorganization of primary auditory cortex, suggesting that NBM plays an important role in representational plasticity, by emphasizing behaviorally important stimuli, which may contribute to improved memory acquisition by placing increased importance on specific stimuli..$^{50}$ The efficacy of DBS of the NBM depends more on frequency than current density, probably because NBM neurons typically have a low-frequency firing rate, and low-frequency stimulation is believed to activate cell bodies. ${ }^{24}$

Unilateral NBM stimulation in rats increased ipsilateral cortical nerve growth factor levels, ${ }^{41}$ and has thus been postulated to potentially mediate neuroprotective effects in AD. This effect is modulated by cholinergic cortical input, and was observed in adult but not aged rats. ${ }^{40}$ Nerve growth factor is important for maintenance and survival of NBM neurons, and is decreased in the NBM in AD. ${ }^{38}$

Deep brain stimulation of the left NBM was performed in a patient with $\mathrm{AD}$, with a frequency of $50 \mathrm{~Hz}$ delivered for 15 seconds on, followed by 12 minutes off. Although no changes in cognitive function were observed, an arrest in the decline of cortical metabolic activity on the side of stimulation was observed. ${ }^{107}$ A clinical trial of DBS of the NBM for AD is currently under way. 33,34

Low-frequency bilateral stimulation of NBM resulted in significant cognitive gains in a patient with severe PDrelated dementia, perhaps via activation of ascending basal forebrain projections and enhancement of the remaining function of the nucleus. ${ }^{22}$ Stabilization was maintained at 2-year follow-up, and the authors postulate that this may have been mediated by stimulation-induced release of nerve growth factor in the NBM as well as resetting of neural oscillations. ${ }^{33}$ Augmentation of cortical cholinergic function by NBM stimulation is a promising target for memory disorders. 


\section{Midbrain Raphe Nuclei}

The dorsal and median raphe nuclei are serotonergic midbrain nuclei that send projections to forebrain gray matter, diencephalon, and brainstem. The median raphe nucleus projects to the dorsal hippocampus. Stimulation of the median raphe nucleus in a rat model of TBI improved function on memory tasks, resulted in increased volume of the parietal occipital cortex (which was found to be decreased in injured rats), and restored adenosine $3^{\prime}, 5^{\prime}$-cyclic monophosphate (cAMP) levels in cortex and hippocampus, but did not affect these parameters in sham-treated animals. $^{8}$

\section{Pedunculopontine Nucleus}

The pedunculopontine nucleus (PPN) modulates activity of frontostriatal circuits and may alter cognitive processing. Low-frequency DBS of the PPN in patients with PD decreased reaction time but did not affect accuracy on a working memory task. Motor reaction times were not altered, suggesting an effect on working memory efficiency, perhaps through alteration of corticostriatal working memory circuits given known strong connections between PPN and basal ganglia, or via increased attention allocation. ${ }^{10}$ In a group of 6 patients with PD who underwent DBS of both subthalamic nucleus (STN) and PPN, low-frequency PPN stimulation resulted in increased metabolism in the inferior frontal gyrus, dorsolateral prefrontal cortex, orbitofrontal cortex, anterior cingulate cortex, superior frontal gyrus, inferior parietal lobule, and supramarginal gyrus as well as the left ventral striatum, right insula, and right superior temporal gyrus. Deep brain stimulation of the PPN was also associated with improved cognitive functions, including verbal long-term memory. ${ }^{99}$

\section{Basal Ganglia}

The basal ganglia associative prefrontal and limbic loops contribute to memory formation and recall, ${ }^{43}$ and therefore neuromodulation of these structures may impact memory.

Approximately $30 \%$ of patients with PD ultimately develop dementia, with cortical and subcortical Lewy bodies and cholinergic degeneration. ${ }^{22}$ Cognitive deficits may be related to reduced dopamine in frontostriatal projections. ${ }^{10}$ Dementia related to PD is characterized by deficits in executive function and memory, and probably occurs via a combination of basal ganglia as well as cortical and other subcortical degenerative processes. ${ }^{24}$

The STN is frequently targeted with DBS to improve the motor symptoms of PD. Reports of effects on cognition in these patients are mixed. Patients with PD have impairments in associative learning and visual working memory that are partially restored by DBS of the STN..$^{2}$ Deep brain stimulation of the STN improves motor sequence learning in patients with PD, and is associated with increased network activity measured during learning, increased activity in the left cerebellar hemisphere and right premotor cortex, and decreased activity in the orbitofrontal cortex and supplementary motor area. ${ }^{75}$ However, DBS of the STN may impair verbal memory in patients with PD. ${ }^{59}$ The mixed effects of DBS of the STN on learning in patients with PD may be related to the different types of learning measured and the variations in lead placement optimized for impact on motor rather than cognitive circuitry.

High-frequency stimulation of the caudate nucleus in primates during the reinforcement period of a visuomotor associative learning task increased the rate of learning, suggesting that the caudate is involved in adjusting associative weights between stimuli and responses. ${ }^{112}$ Striatal neurons have dopaminergic input and demonstrate different degrees of response to rewards, and therefore may contribute to forming learned associations. ${ }^{43}$ Stimulation of the left dorsal striatum in rats immediately after training resulted in rats making significantly more procedural errors on probe tests, and increased GABA in dorsal striatum..$^{90}$ Different timing of stimulation with respect to components of the learning task probably contributed to these differing results, suggesting that the beneficial effect of caudate stimulation on learning is related to increased valuation of reinforcement.

\section{White Matter Targets \\ Fornix}

Forniceal stimulation has been shown to restore memory in rodent models of a number of memory disorders. A rat dementia model created by injecting animals with scopolamine, a muscarinic acetylcholine antagonist, had improved performance on a spatial memory task with forniceal DBS when the animal was stimulated during the task, and this effect was dependent on current levels but not stimulation frequency. ${ }^{39}$ In rats treated with hippocampal A-beta 1-42 injection to simulate AD, high-frequency stimulation of the anterior nucleus of the thalamus, entorhinal cortex, and fornix all improved performance on a spatial memory test, with the greatest improvements found in rats with entorhinal cortex and fornix stimulation. Entorhinal cortex and fornix high-frequency stimulation also improved recognition memory. ${ }^{113}$ The dependence of forniceal DBS effects on current density rather than frequency of stimulation may be because myelinated axons are activated by stimulation regardless of frequency. ${ }^{38}$

High-frequency stimulation of the fornix in mice with Rett syndrome rescued spatial learning, and it improved spatial learning in wild-type mice. Forniceal high-frequency stimulation was associated with improved dentate gyrus long-term potentiation and increased dentate gyrus neurogenesis. ${ }^{32}$

The effects of forniceal DBS may occur via both orthodromic and antidromic activation of the large myelinated axons of the fornix. ${ }^{34,38}$ Deep brain stimulation of the fornix increases a marker of neuronal activity in the dentate gyrus granule cell layer as well as CA1 and CA3. Forniceal DBS also increased hippocampal expression of the neurotrophic factors brain-derived neurotrophic factor and vascular endothelial growth factor, as well as synaptic proteins GAP-43, synaptophysin, and alpha synuclein. ${ }^{23}$ These changes in gene expression may represent neuroprotective mechanisms, increased neurogenesis, and increased synaptic plasticity, including long-term potentiation induced by DBS.

The supramammillary area provides input to the medial septal area that helps determine hippocampal theta rhythmogenicity. ${ }^{68}$ In a rat model in which hippocampal 
theta activity was blocked by a deactivating injection into the medial septal area, stimulation of the fornix at a frequency determined from a recording obtained in the supramammillary area as well as theta range stimulation, but not irregular stimulation, during a spatial memory task resulted in restoration of hippocampal theta activity and restoration of learning in the spatial memory task. ${ }^{68}$ Theta burst stimulation prior to or during learning, but not highor low-frequency constant stimulation delivered via a fornix electrode, improved memory in rats with TBI, perhaps via long-term potentiation-mediated synaptic plasticity. ${ }^{105}$ Specific forniceal stimulation parameters may be able to bypass dysfunctional hippocampal activity and thereby improve memory function by restoring the hippocampal firing rhythms necessary for memory.

Forniceal DBS has also shown promise in human subjects. In epilepsy patients with implanted depth electrodes, low-frequency stimulation of the fornix caused evoked responses in the hippocampus and posterior cingulate gyrus, and was associated with improvement in repeated MiniMental State Examination (MMSE) scores. ${ }^{53}$ A blinded, sham-controlled trial of stimulation of an electrode implanted in the unilateral crus of the fornix in 4 patients with epilepsy undergoing depth electrode placement found that theta burst stimulation of the fornix led to evoked potentials in the hippocampus and improved performance on a spatial memory task, but not on verbal memory or naming, which are types of semantic memory. ${ }^{71}$

The fornix was chosen as the target for the first clinical trial of DBS in AD. Six patients with early AD underwent bilateral DBS with lead placement parallel to the vertical portion of the fornix in the hypothalamus. At 12 months, 2 patients had less than expected worsening in cognitive score, 1 had greater worsening than expected, and 3 had expected worsening in cognitive score. Improvements were largely related to changes in the recall and recognition subscale. Stimulation was associated with changes in activity of the hippocampus and parahippocampal gyrus as well as activation of the middle and posterior cingulate gyrus and precuneus. Increased metabolism was seen in temporal, parietal, and posterior cingulate regions, areas affected in AD, after 1 year of stimulation. ${ }^{56}$ Two patients had an increase in hippocampal volume at 1 year, which correlated with cognitive outcomes; these same patients also had an increase in hippocampal metabolism. Stimulation was also associated with volume increases in other regions involved in AD, including bilateral parahippocampal gyrus, right superior temporal gyrus, left inferior parietal lobule, and bilateral precuneus. ${ }^{88}$ Patients had increased metabolism in 2 networks - a precuneus network that has connections with anterior cingulate and frontal cortical regions as well as limited temporal regions, and a hippocampal/parahippocampal gyrus network with extensive parietal and visual association cortex connections-after 1 year of DBS. Increased metabolism in the anterior cingulate, frontal lobe, insula, superior and middle temporal gyrus, precuneus, posterior cingulate, and cerebellum at 1 year correlated with cognitive scores, as did decreased metabolism in the precentral gyrus, middle occipital gyrus, putamen, and pulvinar. ${ }^{95}$ Stimulation was more effective in patients with better preoperative function, perhaps be- cause in early AD the hippocampus-to-fornix connections may still be functionally intact and therefore better able to have function augmented. ${ }^{34}$ In another study a single patient with bilateral forniceal DBS had stabilization of cognitive and memory scores and increased mesial temporal lobe metabolism at 1-year follow-up. ${ }^{20}$

Overall, these results support the model that forniceal DBS activates the fornix and alters upstream and downstream signaling in memory circuitry, creating changes in metabolism and structure via alteration of network signaling and perhaps neurogenesis. Neurogenesis induced by DBS may contribute new neurons that integrate into functional circuits and improve function in the neuronal network underlying memory. ${ }^{62}$ Although studies of forniceal DBS in humans have demonstrated promising metabolic changes in networks associated with memory, further studies are needed to determine whether these will be associated with durable clinical results.

\section{Medial Forebrain Bundle}

In rodents, intracranial self-stimulation of the medial forebrain bundle administered after training sessions improves learning, ${ }^{48,97}$ reinforcing learning from the previous session. ${ }^{86}$ Intracranial self-stimulation administered after memory reactivation improved retrieval when tested 24 hours later but not immediately after stimulation, suggesting long-term facilitation of memory retrieval. ${ }^{98}$

Intracranial self-stimulation potentiates long-term memory ${ }^{1}$ and increases a marker of neuronal activity in basolateral amygdala, lateral amygdala $,{ }^{48} \mathrm{CA} 1, \mathrm{CA} 3$, dentate gyrus, dorsal striatum, and lateral hypothalamus. It also modulates expression of genes involved in learning and memory and in neural plasticity and neurogenesis in the hippocampus. ${ }^{44}$ Expression of immediate early gene Nurrl, a transcription factor expressed in dopaminergic cells, was significantly increased in CA1, CA3, dentate gyrus, dorsal striatum, and lateral hypothalamus after intracranial self-stimulation. ${ }^{1}$ Stimulation upregulates expression of genes involved in neurogenesis in the hippocampus. ${ }^{34}$ Expression of genes involved in synaptic plasticity, protein folding, and neurogenesis was increased in the amygdala after intracranial self-stimulation. ${ }^{48}$ The time delay requirement of stimulation after encoding, combined with changes in expression of genes involved in protein folding and neural plasticity and neurogenesis, supports augmented plasticity and perhaps neurogenesis in limbic circuitry as the mechanism of action of medial forebrain bundle DBS in memory.

\section{Other Therapies}

Transcranial electrical nerve stimulation has been associated with memory improvement in patients with AD, and may act via hippocampal activation. ${ }^{26,31,89}$

Radioelectrical asymmetrical brain stimulation is a noninvasive therapy using radiofrequency bursts. It has been shown to improve MMSE scores in AD, although longevity of results was not demonstrated. ${ }^{64}$

Vagal nerve stimulation may improve cognitive outcome in patients with $\mathrm{AD}$, with cognitive scale scores stable or improved at 6 months in $70 \%$ of patients. ${ }^{94}$ At 
1-year follow-up, $41 \%$ had improvement or no change on the Alzheimer's Disease Assessment Scale-cognitive subscale (ADAS-cog) and $71 \%$ had improvement or no change on the MMSE. ${ }^{69}$ Vagal nerve stimulation may also improve cognitive function in TBI. Putative mechanisms include increased norepinephrine via activation of the locus coeruleus, antiinflammatory effect, increased synaptic plasticity, and perhaps increased neurogenesis. ${ }^{76}$

\section{Conclusions}

Rapid advances in mapping the neural circuitry underlying learning and memory in both rodent models and human patients over the past 20 years have resulted in a proliferation of potential therapeutic targets for neuromodulation. As reviewed above, manipulating many of these targets has shown therapeutic promise in mouse and rat models of TBI and AD. Initial clinical trials targeting the fornix have had mixed results. Longer-term outcomes data of the various investigated targets in humans are particularly sparse. Additionally, although the discussed therapeutics have shown promise in improving memory in TBI and AD, it is important to acknowledge that both TBI and AD are disorders of overall cognition and not solely memory; thus it is difficult to determine if improving memory will translate into gains in quality of life. The data are compelling in support of further experimentation aimed at additional targets including entorhinal cortex, NBM, and PPN. Stimulation of different targets probably affects memory via a number of mechanisms, including alteration of neural firing patterns important for memory encoding and retrieval, augmentation of neural plasticity, and neurogenesis. The most effective strategies will probably require more sophisticated stimulated parameters and strategies and potentially multiple targets, with the ultimate goal of creating a responsive closed-loop system. The clinical burden of dementia is considerable and the efficacy of current medical treatments is limited, so significant further investigation is well warranted.

\section{References}

1. Aldavert-Vera L, Huguet G, Costa-Miserachs D, Ortiz SP, Kádár E, Morgado-Bernal I, et al: Intracranial self-stimulation facilitates active-avoidance retention and induces expression of c-Fos and Nurr1 in rat brain memory systems. Behav Brain Res 250:46-57, 2013

2. Arrieta-Cruz I, Pavlides C, Pasinetti GM: Deep brain stimulation in midline thalamic region facilitates synaptic transmission and shortterm memory in a mouse model of Alzheimer's disease. Transl Neurosci 1:188-194, 2010

3. Bari A, Niu T, Langevin JP, Fried I: Limbic neuromodulation: implications for addiction, posttraumatic stress disorder, and memory. Neurosurg Clin N Am 25:137-145, 2014

4. Berger T, Song D, Chan R, Shin D, Marmarelis V, Hampson $\mathrm{R}$, et al: Role of the hippocampus in memory formation: restorative encoding memory integration neural device as a cognitive neural prosthesis. IEEE Pulse 3:17-22, 2012

5. Berger TW, Hampson RE, Song D, Goonawardena A, Marmarelis VZ, Deadwyler SA: A cortical neural prosthesis for restoring and enhancing memory. J Neural Eng 8:046017, 2011

6. Boggio PS, Ferrucci R, Mameli F, Martins D, Martins O, Vergari M, et al: Prolonged visual memory enhancement after direct current stimulation in Alzheimer's disease. Brain Stimulat 5:223-230, 2012
7. Boix-Trelis N, Vale-Martínez A, Guillazo-Blanch G, CostaMiserachs D, Martí-Nicolovius M: Effects of nucleus basalis magnocellularis stimulation on a socially transmitted food preference and c-Fos expression. Learn Mem 13:783-793, 2006

8. Carballosa Gonzalez MM, Blaya MO, Alonso OF, Bramlett HM, Hentall ID: Midbrain raphe stimulation improves behavioral and anatomical recovery from fluid-percussion brain injury. J Neurotrauma 30:119-130, 2013

9. Chen N, Dong S, Yan T, Yan N, Ma Y, Yu C: Highfrequency stimulation of anterior nucleus thalamus improves impaired cognitive function induced by intra-hippocampal injection of A $\beta 1-40$ in rats. Chin Med J (Engl) 127:125129, 2014

10. Costa A, Carlesimo GA, Caltagirone C, Mazzone P, Pierantozzi M, Stefani A, et al: Effects of deep brain stimulation of the peduncolopontine area on working memory tasks in patients with Parkinson's disease. Parkinsonism Relat Disord 16:64-67, 2010

11. Cotelli M, Calabria M, Manenti R, Rosini S, Maioli C, Zanetti $\mathrm{O}$, et al: Brain stimulation improves associative memory in an individual with amnestic mild cognitive impairment. Neurocase 18:217-223, 2012

12. de Souza Custódio JC, Martins CW, Lugon MD, Fregni F, Nakamura-Palacios EM: Epidural direct current stimulation over the left medial prefrontal cortex facilitates spatial working memory performance in rats. Brain Stimulat 6:261-269, 2013

13. Deep-Brain Stimulation for Parkinson's Disease Study Group: Deep-brain stimulation of the subthalamic nucleus or the pars interna of the globus pallidus in Parkinson's disease. N Engl J Med 345:956-963, 2001

14. Dhaliwal SK, Meek BP, Modirrousta MM: Non-invasive brain stimulation for the treatment of symptoms following traumatic brain injury. Front Psychiatry 6:119, 2015

15. Encinas JM, Hamani C, Lozano AM, Enikolopov G: Neurogenic hippocampal targets of deep brain stimulation. J Comp Neurol 519:6-20, 2011

16. Fell J, Axmacher N: The role of phase synchronization in memory processes. Nat Rev Neurosci 12:105-118, 2011

17. Fell J, Klaver P, Lehnertz K, Grunwald T, Schaller C, Elger $\mathrm{CE}$, et al: Human memory formation is accompanied by rhinal-hippocampal coupling and decoupling. Nat Neurosci 4:1259-1264, 2001

18. Fell J, Staresina BP, Do Lam AT, Widman G, Helmstaedter $C$, Elger CE, et al: Memory modulation by weak synchronous deep brain stimulation: a pilot study. Brain Stimulat 6:270-273, 2013

19. Fisher R, Salanova V, Witt T, Worth R, Henry T, Gross R, et al: Electrical stimulation of the anterior nucleus of thalamus for treatment of refractory epilepsy. Epilepsia 51:899-908, 2010

20. Fontaine D, Deudon A, Lemaire JJ, Razzouk M, Viau P, Darcourt J, et al: Symptomatic treatment of memory decline in Alzheimer's disease by deep brain stimulation: a feasibility study. J Alzheimers Dis 34:315-323, 2013

21. Fregni F, Boggio PS, Nitsche M, Bermpohl F, Antal A, Feredoes E, et al: Anodal transcranial direct current stimulation of prefrontal cortex enhances working memory. Exp Brain Res 166:23-30, 2005

22. Freund HJ, Kuhn J, Lenartz D, Mai JK, Schnell T, Klosterkoetter J, et al: Cognitive functions in a patient with Parkinson-dementia syndrome undergoing deep brain stimulation. Arch Neurol 66:781-785, 2009

23. Gondard E, Chau HN, Mann A, Tierney TS, Hamani C, Kalia SK, et al: Rapid modulation of protein expression in the rat hippocampus following deep brain stimulation of the fornix. Brain Stimulat 8: 1058-1064, 2015

24. Gratwicke J, Kahan J, Zrinzo L, Hariz M, Limousin P, 
Foltynie T, et al: The nucleus basalis of Meynert: a new target for deep brain stimulation in dementia? Neurosci Biobehav Rev 37:2676-2688, 2013

25. Greenberg BD, Malone DA, Friehs GM, Rezai AR, Kubu CS, Malloy PF, et al: Three-year outcomes in deep brain stimulation for highly resistant obsessive-compulsive disorder. Neuropsychopharmacology 31:2384-2393, 2006

26. Guo Y, Shi X, Uchiyama H, Hasegawa A, Nakagawa Y, Tanaka M, et al: A study on the rehabilitation of cognitive function and short-term memory in patients with Alzheimer's disease using transcutaneous electrical nerve stimulation. Front Med Biol Eng 11:237-247, 2002

27. Hamani C, Dubiela FP, Soares JC, Shin D, Bittencourt S, Covolan L, et al: Anterior thalamus deep brain stimulation at high current impairs memory in rats. Exp Neurol 225:154-162, 2010

28. Hamani C, McAndrews MP, Cohn M, Oh M, Zumsteg D, Shapiro CM, et al: Memory enhancement induced by hypothalamic/fornix deep brain stimulation. Ann Neurol 63: $119-123,2008$

29. Hamani C, Stone SS, Garten A, Lozano AM, Winocur G: Memory rescue and enhanced neurogenesis following electrical stimulation of the anterior thalamus in rats treated with corticosterone. Exp Neurol 232:100-104, 2011

30. Hånell A, Greer JE, Jacobs KM: Increased network excitability due to altered synaptic inputs to neocortical layer $\mathrm{v}$ intact and axotomized pyramidal neurons after mild traumatic brain injury. J Neurotrauma 32:1590-1598, 2015

31. Hansen N: Brain stimulation for combating Alzheimer's disease. Front Neurol 5:80, 2014

32. Hao S, Tang B, Wu Z, Ure K, Sun Y, Tao H, et al: Forniceal deep brain stimulation rescues hippocampal memory in Rett syndrome mice. Nature 526:430-434, 2015

33. Hardenacke K, Kuhn J, Lenartz D, Maarouf M, Mai JK, Bartsch C, et al: Stimulate or degenerate: deep brain stimulation of the nucleus basalis Meynert in Alzheimer dementia. World Neurosurg 80:S27.e35-S27.e43, 2013

34. Hardenacke K, Shubina E, Bührle CP, Zapf A, Lenartz D, Klosterkötter J, et al: Deep brain stimulation as a tool for improving cognitive functioning in Alzheimer's dementia: a systematic review. Front Psychiatry 4:159, 2013

35. Hartikainen KM, Sun L, Polvivaara M, Brause M, Lehtimäki K, Haapasalo J, et al: Immediate effects of deep brain stimulation of anterior thalamic nuclei on executive functions and emotion-attention interaction in humans. J Clin Exp Neuropsychol 36:540-550, 2014

36. Hebert LE, Weuve J, Scherr PA, Evans DA: Alzheimer disease in the United States (2010-2050) estimated using the 2010 census. Neurology 80:1778-1783, 2013

37. Hescham S, Jahanshahi A, Meriaux C, Lim LW, Blokland A, Temel Y: Behavioral effects of deep brain stimulation of different areas of the Papez circuit on memory- and anxietyrelated functions. Behav Brain Res 292:353-360, 2015

38. Hescham S, Lim LW, Jahanshahi A, Blokland A, Temel Y: Deep brain stimulation in dementia-related disorders. Neurosci Biobehav Rev 37:2666-2675, 2013

39. Hescham S, Lim LW, Jahanshahi A, Steinbusch HW, Prickaerts J, Blokland A, et al: Deep brain stimulation of the forniceal area enhances memory functions in experimental dementia: the role of stimulation parameters. Brain Stimulat 6:72-77, 2013

40. Hotta H, Kagitani F, Kondo M, Uchida S: Basal forebrain stimulation induces NGF secretion in ipsilateral parietal cortex via nicotinic receptor activation in adult, but not aged rats. Neurosci Res 63:122-128, 2009

41. Hotta H, Uchida S, Kagitani F: Stimulation of the nucleus basalis of Meynert produces an increase in the extracellular release of nerve growth factor in the rat cerebral cortex. $\mathbf{J}$ Physiol Sci 57:383-387, 2007
42. Houeto JL, Karachi C, Mallet L, Pillon B, Yelnik J, Mesnage V, et al: Tourette's syndrome and deep brain stimulation. J Neurol Neurosurg Psychiatry 76:992-995, 2005

43. Hu R, Eskandar E, Williams Z: Role of deep brain stimulation in modulating memory formation and recall. Neurosurg Focus 27(1):E3, 2009

44. Huguet G, Aldavert-Vera L, Kádár E, Peña de Ortiz S, Morgado-Bernal I, Segura-Torres P: Intracranial self-stimulation to the lateral hypothalamus, a memory improving treatment, results in hippocampal changes in gene expression. Neuroscience 162:359-374, 2009

45. Jaušovec $N$, Jaušovec $K$ : Increasing working memory capacity with theta transcranial alternating current stimulation (tACS). Biol Psychol 96:42-47, 2014

46. Javadi AH, Walsh V: Transcranial direct current stimulation (tDCS) of the left dorsolateral prefrontal cortex modulates declarative memory. Brain Stimulat 5:231-241, 2012

47. Jeong da U: Lee JE, Lee SE, Chang WS, Kim SJ, Chang JW: Improvements in memory after medial septum stimulation are associated with changes in hippocampal cholinergic activity and neurogenesis. BioMed Res Int 2014:568587, 2014

48. Kadar E, Aldavert-Vera L, Huguet G, Costa-Miserachs D, Morgado-Bernal I, Segura-Torres P: Intracranial self-stimulation induces expression of learning and memory-related genes in rat amygdala. Genes Brain Behav 10:69-77, 2011

49. Kádár E, Ramoneda M, Aldavert-Vera L, Huguet G, Morgado-Bernal I, Segura-Torres P: Rewarding brain stimulation reverses the disruptive effect of amygdala damage on emotional learning. Behav Brain Res 274:43-52, 2014

50. Kilgard MP, Merzenich MM: Cortical map reorganization enabled by nucleus basalis activity. Science 279:1714-1718, 1998

51. Köhler S, Paus T, Buckner RL, Milner B: Effects of left inferior prefrontal stimulation on episodic memory formation: a two-stage fMRI-rTMS study. J Cogn Neurosci 16:178-188, 2004

52. Koski L, Kolivakis T, Yu C, Chen JK, Delaney S, Ptito A: Noninvasive brain stimulation for persistent postconcussion symptoms in mild traumatic brain injury. $\mathbf{J}$ Neurotrauma 32:38-44, 2015

53. Koubeissi MZ, Kahriman E, Syed TU, Miller J, Durand DM: Low-frequency electrical stimulation of a fiber tract in temporal lobe epilepsy. Ann Neurol 74:223-231, 2013

54. Lacruz ME, Valentín A, Seoane JJ, Morris RG, Selway RP, Alarcón G: Single pulse electrical stimulation of the hippocampus is sufficient to impair human episodic memory. Neuroscience 170:623-632, 2010

55. Laxton AW, Lipsman N, Lozano AM: Deep brain stimulation for cognitive disorders. Handb Clin Neurol 116:307311,2013

56. Laxton AW, Tang-Wai DF, McAndrews MP, Zumsteg D, Wennberg R, Keren R, et al: A phase I trial of deep brain stimulation of memory circuits in Alzheimer's disease. Ann Neurol 68:521-534, 2010

57. Lee DJ, Gurkoff GG, Izadi A, Berman RF, Ekstrom AD, Muizelaar JP, et al: Medial septal nucleus theta frequency deep brain stimulation improves spatial working memory after traumatic brain injury. J Neurotrauma 30:131-139, 2013

58. Lee DJ, Gurkoff GG, Izadi A, Seidl SE, Echeverri A, Melnik M, et al: Septohippocampal neuromodulation improves cognition after traumatic brain injury. $\mathbf{J}$ Neurotrauma 32: 1822-1832, 2015

59. Lee H, Fell J, Axmacher N: Electrical engram: how deep brain stimulation affects memory. Trends Cogn Sci 17:574-584, 2013

60. Liu A, Jain N, Vyas A, Lim LW: Ventromedial prefrontal cortex stimulation enhances memory and hippocampal neurogenesis in the middle-aged rats. eLife 4:4, 2015 
61. Liu X, Ramirez S, Pang PT, Puryear CB, Govindarajan A, Deisseroth K, et al: Optogenetic stimulation of a hippocampal engram activates fear memory recall. Nature 484:381385,2012

62. Lyketsos CG, Targum SD, Pendergrass JC, Lozano AM: Deep brain stimulation: a novel strategy for treating Alzheimer's disease. Innov Clin Neurosci 9: 10-17, 2012

63. Mair RG, Hembrook JR: Memory enhancement with eventrelated stimulation of the rostral intralaminar thalamic nuclei. J Neurosci 28: 14293-14300, 2008

64. Mannu P, Rinaldi S, Fontani V, Castagna A: Radio electric asymmetric brain stimulation in the treatment of behavioral and psychiatric symptoms in Alzheimer disease. Clin Interv Aging 6:207-211, 2011

65. Mayberg HS, Lozano AM, Voon V, McNeely HE, Seminowicz D, Hamani C, et al: Deep brain stimulation for treatment-resistant depression. Neuron 45:651-660, 2005

66. McLachlan RS, Pigott S, Tellez-Zenteno JF, Wiebe S, Parrent A: Bilateral hippocampal stimulation for intractable temporal lobe epilepsy: impact on seizures and memory. Epilepsia 51:304-307, 2010

67. McLin DE III, Miasnikov AA, Weinberger NM: Induction of behavioral associative memory by stimulation of the nucleus basalis. Proc Natl Acad Sci U S A 99:4002-4007, 2002

68. McNaughton N, Ruan M, Woodnorth MA: Restoring thetalike rhythmicity in rats restores initial learning in the Morris water maze. Hippocampus 16:1102-1110, 2006

69. Merrill CA, Jonsson MA, Minthon L, Ejnell H, C-son Silander H, Blennow K, et al: Vagus nerve stimulation in patients with Alzheimer's disease: Additional follow-up results of a pilot study through 1 year. J Clin Psychiatry 67:1171-1178, 2006

70. Miatton M, Van Roost D, Thiery E, Carrette E, Van Dycke A, Vonck K, et al: The cognitive effects of amygdalohippocampal deep brain stimulation in patients with temporal lobe epilepsy. Epilepsy Behav 22:759-764, 2011

71. Miller JP, Sweet JA, Bailey CM, Munyon CN, Luders HO, Fastenau PS: Visual-spatial memory may be enhanced with theta burst deep brain stimulation of the fornix: a preliminary investigation with four cases. Brain 138:1833-1842, 2015

72. Mollion H, Dominey PF, Broussolle E, Ventre-Dominey J: Subthalamic nucleus stimulation selectively improves motor and visual memory performance in Parkinson's disease. Mov Disord 26:2019-2025, 2011

73. Montero-Pastor A, Vale-Martínez A, Guillazo-Blanch G, Martí-Nicolovius M: Effects of electrical stimulation of the nucleus basalis on two-way active avoidance acquisition, retention, and retrieval. Behav Brain Res 154:41-54, 2004

74. Montero-Pastor A, Vale-Martínez A, Guillazo-Blanch G, Nadal-Alemany R, Martí-Nicolovius M, Morgado-Bernal I: Nucleus basalis magnocellularis electrical stimulation facilitates two-way active avoidance retention, in rats. Brain Res 900:337-341, 2001

75. Mure H, Tang CC, Argyelan M, Ghilardi MF, Kaplitt MG, Dhawan V, et al: Improved sequence learning with subthalamic nucleus deep brain stimulation: evidence for treatment-specific network modulation. J Neurosci 32:2804-2813, 2012

76. Neren D, Johnson MD, Legon W, Bachour SP, Ling G, Divani AA: Vagus nerve stimulation and other neuromodulation methods for treatment of traumatic brain injury. Neurocrit Care [epub ahead of print], 2015

77. Oh YS, Kim HJ, Lee KJ, Kim YI, Lim SC, Shon YM: Cognitive improvement after long-term electrical stimulation of bilateral anterior thalamic nucleus in refractory epilepsy patients. Seizure 21:183-187, 2012

78. Ojemann GA, Blick KI, Ward AA Jr: Improvement and disturbance of short-term verbal memory with human ventrolateral thalamic stimulation. Brain 94:225-240, 1971

79. Pachalska M, Łukowicz M, Kropotov JD, HermanSucharska I, Talar J: Evaluation of differentiated neurotherapy programs for a patient after severe TBI and long term coma using event-related potentials. Med Sci Monit 17:CS120-CS128, 2011

80. Papez JW: A proposed mechanism of emotion. 1937. J Neuropsychiatry Clin Neurosci 7:103-112, 1995

81. Parikh S, Koch M, Narayan RK: Traumatic brain injury. Int Anesthesiol Clin 45:119-135, 2007

82. Paterno R, Metheny H, Xiong G, Elkind J, Cohen AS: Mild traumatic brain injury decreases broadband power in area CA1. J Neurotrauma [epub ahead of print], 2016

83. Pereira JL, Downes A, Gorgulho A, Patel V, Malkasian D, De Salles A: Alzheimer's disease: The role for neurosurgery. Surg Neurol Int 5 (Suppl 8):S385-S390, 2014

84. Plassman BL, Langa KM, Fisher GG, Heeringa SG, Weir DR, Ofstedal MB, et al: Prevalence of dementia in the United States: the aging, demographics, and memory study. Neuroepidemiology 29:125-132, 2007

85. Rasmusson DD, Clow K, Szerb JC: Frequency-dependent increase in cortical acetylcholine release evoked by stimulation of the nucleus basalis magnocellularis in the rat. Brain Res 594:150-154, 1992

86. Ruiz-Medina J, Redolar-Ripoll D, Morgado-Bernal I, Aldavert-Vera L, Segura-Torres P: Intracranial self-stimulation improves memory consolidation in rats with little training. Neurobiol Learn Mem 89:574-581, 2008

87. Salviati M, Bersani FS, Calabria LF, Rapinesi C, Kotzalidis GD, Minichino A, et al: Deep transcranial magnetic stimulation in a woman with chronic tinnitus: clinical and FMRI findings. Seeking relief from a symptom and finding vivid memories by serendipity. Brain Stimulat 7:492-494, 2014

88. Sankar T, Chakravarty MM, Bescos A, Lara M, Obuchi T, Laxton AW, et al: Deep brain stimulation influences brain structure in Alzheimer's disease. Brain Stimulat 8:645654,2015

89. Scherder EJ, Bouma A, Steen LM: Effects of "isolated" transcutaneous electrical nerve stimulation on memory and affective behavior in patients with probable Alzheimer's disease. Biol Psychiatry 43:417-424, 1998

90. Schumacher A, de Vasconcelos AP, Lecourtier L, Moser A, Cassel JC: Electrical high frequency stimulation in the dorsal striatum: Effects on response learning and on GABA levels in rats. Behav Brain Res 222:368-374, 2011

91. Schuurman PR, Bosch DA, Bossuyt PM, Bonsel GJ, van Someren EJ, de Bie RM, et al: A comparison of continuous thalamic stimulation and thalamotomy for suppression of severe tremor. N Engl J Med 342:461-468, 2000

92. Sharma M, Deogaonkar M, Rezai A: Assessment of potential targets for deep brain stimulation in patients with Alzheimer's disease. J Clin Med Res 7:501-505, 2015

93. Shirvalkar P, Seth M, Schiff ND, Herrera DG: Cognitive enhancement with central thalamic electrical stimulation. Proc Natl Acad Sci U S A 103:17007-17012, 2006

94. Sjögren MJ, Hellström PT, Jonsson MA, Runnerstam M, Silander HC, Ben-Menachem E: Cognition-enhancing effect of vagus nerve stimulation in patients with Alzheimer's disease: a pilot study. J Clin Psychiatry 63:972-980, 2002

95. Smith GS, Laxton AW, Tang-Wai DF, McAndrews MP, Diaconescu AO, Workman CI, et al: Increased cerebral metabolism after 1 year of deep brain stimulation in Alzheimer disease. Arch Neurol 69:1141-1148, 2012

96. Solé-Padullés C, Bartrés-Faz D, Junqué C, Clemente IC, Molinuevo JL, Bargalló N, et al: Repetitive transcranial magnetic stimulation effects on brain function and cognition among elders with memory dysfunction. A randomized sham-controlled study. Cereb Cortex 16:1487-1493, 2006 
97. Soriano-Mas C, Redolar-Ripoll D, Aldavert-Vera L, Morgado-Bernal I, Segura-Torres P: Post-training intracranial self-stimulation facilitates a hippocampus-dependent task. Behav Brain Res 160:141-147, 2005

98. Soriano-Mas C, Redolar-Ripoll D, Guillazo-Blanch G, Morgado-Bernal I, Segura-Torres P: Intracranial self-stimulation after memory reactivation: immediate and late effects. Brain Res Bull 74:51-57, 2007

99. Stefani A, Pierantozzi M, Ceravolo R, Brusa L, Galati S, Stanzione P: Deep brain stimulation of pedunculopontine tegmental nucleus (PPTg) promotes cognitive and metabolic changes: a target-specific effect or response to a low-frequency pattern of stimulation? Clin EEG Neurosci 41:82-86, 2010

100. Stone SS, Teixeira CM, Devito LM, Zaslavsky K, Josselyn SA, Lozano AM, et al: Stimulation of entorhinal cortex promotes adult neurogenesis and facilitates spatial memory. J Neurosci 31:13469-13484, 2011

101. Stone SS, Teixeira CM, Zaslavsky K, Wheeler AL, Martinez-Canabal A, Wang AH, et al: Functional convergence of developmentally and adult-generated granule cells in dentate gyrus circuits supporting hippocampus-dependent memory. Hippocampus 21:1348-1362, 2011

102. Suthana N, Fried I: Deep brain stimulation for enhancement of learning and memory. Neuroimage 85:996-1002, 2014

103. Suthana N, Haneef Z, Stern J, Mukamel R, Behnke E, Knowlton B, et al: Memory enhancement and deep-brain stimulation of the entorhinal area. N Engl J Med 366:502510,2012

104. Sweeney-Reed CM, Zaehle T, Voges J, Schmitt FC, Buentjen L, Kopitzki K, et al: Corticothalamic phase synchrony and cross-frequency coupling predict human memory formation. eLife 3:e05352, 2014

105. Sweet JA, Eakin KC, Munyon CN, Miller JP: Improved learning and memory with theta-burst stimulation of the fornix in rat model of traumatic brain injury. Hippocampus 24:1592-1600, 2014

106. Toda H, Hamani C, Fawcett AP, Hutchison WD, Lozano AM: The regulation of adult rodent hippocampal neurogenesis by deep brain stimulation. J Neurosurg 108:132-138, 2008

107. Turnbull IM, McGeer PL, Beattie L, Calne D, Pate B: Stimulation of the basal nucleus of Meynert in senile dementia of Alzheimer's type. A preliminary report. Appl Neurophysiol 48:216-221, 1985
108. Ulam F, Shelton C, Richards L, Davis L, Hunter B, Fregni F, et al: Cumulative effects of transcranial direct current stimulation on EEG oscillations and attention/working memory during subacute neurorehabilitation of traumatic brain injury. Clin Neurophysiol 126:486-496, 2015

109. Vidailhet M, Vercueil L, Houeto JL, Krystkowiak P, Benabid AL, Cornu P, et al: Bilateral deep-brain stimulation of the globus pallidus in primary generalized dystonia. $\mathbf{N}$ Engl J Med 352:459-467, 2005

110. Vignal JP, Maillard L, McGonigal A, Chauvel P: The dreamy state: hallucinations of autobiographic memory evoked by temporal lobe stimulations and seizures. Brain 130:88-99, 2007

111. Wang XJ: Pacemaker neurons for the theta rhythm and their synchronization in the septohippocampal reciprocal loop. J Neurophysiol 87:889-900, 2002

112. Williams ZM, Eskandar EN: Selective enhancement of associative learning by microstimulation of the anterior caudate. Nat Neurosci 9:562-568, 2006

113. Zhang C, Hu WH, Wu DL, Zhang K, Zhang JG: Behavioral effects of deep brain stimulation of the anterior nucleus of thalamus, entorhinal cortex and fornix in a rat model of Alzheimer's disease. Chin Med J (Engl) 128:1190-1195, 2015

\section{Disclosures}

The authors report no conflict of interest concerning the materials or methods used in this study or the findings specified in this paper.

\section{Author Contributions}

Conception and design: both authors. Analysis and interpretation of data: Bick. Drafting the article: Bick. Critically revising the article: both authors. Reviewed submitted version of manuscript: both authors. Approved the final version of the manuscript on behalf of both authors: Bick.

\section{Correspondence}

Sarah K. B. Bick, Department of Neurosurgery, Massachusetts General Hospital, 55 Fruit St., Boston, MA 02114. email: sbick@ partners.org. 